\title{
Systemic lupus erythematosus: genetic variants in Xq28 region
}

\author{
Noha A. Doudar ${ }^{1}$, Sanaa S. Abdelshafy ${ }^{1}$, Shaimaa A.K. Rady ${ }^{2}$, Asmaa M. Mokhtar ${ }^{1}$ \\ ${ }^{1}$ Department of Clinical and Chemical Pathology, Faculty of Medicine, Beni-Suef University, Beni-Suef, Egypt \\ ${ }^{2}$ Department of Rheumatology, Faculty of Medicine, Beni-Suef University, Beni-Suef, Egypt
}

\begin{abstract}
Objectives: Methyl-CpG-binding protein 2 (MECP2) and interleukin-1 receptor-associated kinase (IRAK1) are encoded by adjacent $X$-linked genes and recognized for their role in regulation of inflammation. The present case control study was conducted to detect the genetic association between MECP2 (rs1734791) and IRAK1 (rs1059703) single nucleotide polymorphisms (SNPs) and susceptibility to systemic lupus erythematosus (SLE), and the possible association of these SNPs and severity of SLE. Material and methods: Fifty patients with SLE and 100 healthy controls were included in this study. Systemic Lupus International Collaborating Clinics (SLICC) criteria were used to classify SLE patients and the activity of the disease was assessed by SLEDAI score. Disease severity was assessed by the SLICC damage index (SLICC DI). Genetic association of both SNPS with SLE was assessed by Taq Man allelic discrimination technique.

Results: Analyses of MECP2 (rs1734791) SNP genotypes revealed that homozygous TT genotype was significantly higher in the control group than SLE patients $(p<0.001$, odds ratio [OR] $=0.120)$. Frequency of allele (A) was significantly higher in SLE patients, $(p<0.001$, OR $=0.334)$. SLE patients had significantly higher frequency of the homozygous AA and heterozygous AG genotype of IRAK1 (rs1059703) SNP in comparison to healthy controls ( $p=0.0029, \mathrm{OR}=4.17$ and 6.30 respectively). $\mathrm{T}+\mathrm{G}$ and $\mathrm{T}+\mathrm{A}$ of rs1734791 and rs1059703 SNPs are protective haplotypes (OR $=0.47$ and $0.3, p=0.0046$ and $<0.012$ respectively). No significant association between either SNP and disease activity or severity was found. Conclusions: There is a possible genetic association between both rs1734791 and rs1059703 SNPs and susceptibility to SLE, while no significant association between either SNP and disease activity or severity was detected.
\end{abstract}

Key words: systemic lupus erythematosus, inflammation, methyl-CpG-binding protein 2, interleukin 1 receptor-associated kinase.

\section{Introduction}

The pathogenesis of systemic lupus erythematosus (SLE) is complex; mutations in a single gene may result in SLE in only $1-4 \%$ of patients [1], and so genetic predisposition is not enough to develop the full picture of SLE. Instead genetically susceptible individuals develop SLE upon exposure to additional triggering environmental or epigenetic events [2].

Epigenetic mechanisms are heritable events that control gene expression through regulating the accessibility of DNA to the transcriptional complex [3]. Alterations of a number of epigenetic mechanisms including DNA methylation, histone modifications, and non-coding tran- scripts are involved in the dysregulation reported in many autoimmune/inflammatory diseases, including SLE [4].

DNA methylation (adding a methyl group enzymatically via DNA methyltransferase 1 [DNMT1] to the $5^{\prime}$ carbon of cytosine in cytosine-phosphate-guanosine [CpG] dinucleotides) controls the accessibility of regulatory regions of the genes to transcription factors and RNA polymerases [5].

Methyl-CpG-binding protein 2 (MECP2) is a transcriptional regulator that controls the expression of methylation sensitive genes. MECP2 recruits DNMT1 during DNA methylation; it also recruit histone deacetylase complexes to the gene promoter, leading to chromatin conformation and hence silencing of gene expression [6].

Address for correspondence:

Noha A. Doudar, Department of Clinical and Chemical Pathology, Faculty of Medicine, Beni-Suef University, Mokbel 62511,

Beni-Suef, Egypt, e-mail: noha_ad@hotmail.com

Submitted: 3.10.2019; Accepted: 17.10.2019 
MECP2 can also act as a transcriptional activator by recruiting transcription factors, and it may also have a role in regulating RNA splicing [7].

The interleukin 1 receptor-associated kinase 1 (IRAK1) gene is adjacent to the MECP2 gene (separated by $1.7 \mathrm{~kb}$ on $\mathrm{Xq} 28$ ), and both are in high linkage disequilibrium (LD) [8]. IRAK1 is a serine-threonine kinase that acts downstream of the interleukin 1 receptor to up-regulate NF- $\kappa B$, a significant transcription factor involved in the pathogenesis of SLE [9]. IRAK1 is also involved in the Toll-like receptor signaling pathway that activates innate immunity; this mechanism is also participates in the pathogenesis of SLE [10].

Single nucleotide polymorphisms (SNPS) in MECP2 and IRAK1 genes have been independently associated with risk of SLE in Asian and European populations [8, 11-13]. However, similar studies in the Egyptian population are not available yet. So the present study aims to clarify the significance of this association in the Egyptian SLE patients.

\section{Material and methods}

The present study was conducted on 50 SLE patients (47 females and 3 males), diagnosed by a rheumatologist according to Systemic Lupus International Collaborating Clinics classification (SLICC) criteria [14], and 100 age and gender matched healthy unrelated controls with no clinical evidence or family history of any autoimmune diseases. The SLE patients together with the healthy controls were living in the same geographica area and were recruited from the Rheumatology and Rehabilitation Outpatient Clinic in Beni-Suef University Hospital, Egypt. Written informed consent was obtained from all participating patients and controls. The study was approved by the ethical committee of Beni-Suef University in accordance with the Declaration of Helsinki ethical standards (FMBSUREC/06012019/Osman).

\section{Clinical assessment}

All participants in this study were subjected to full history taking, complete clinical examination as well as laboratory assessment for diagnosis and classification of SLE. The Systemic Lupus Erythematosus Disease Activity Index (SLEDAI) score was assessed [15]. A SLEDAI score of $>6$ was considered an active disease. The disease damage was assessed according to the SLICC damage index (SLICC DI) [16].

\section{Genotyping}

Two milliliters of venous blood was withdrawn by a sterile venipuncture into a sterile EDTA vacutainer. Ge- nomic DNA was extracted from whole blood using the QIAamp DNA Mini Kit (QIAGEN) according to the manufacturer's protocol, and stored at $-20^{\circ} \mathrm{C}$.

Genotyping of MECP2 ( $r$ 1734791) (A > T) and IRAK1 (rs1059703) (G > A) SNPs was performed using the MGBTaqMan Allelic Discrimination method (Applied Biosystems, USA). In MECP2 The TaqMan MGB probes/extension primers were VIC ACAAGAAATAAACAGATGATAAAAG to detect the allele 1 sequence and FAM AAAGTATCAGGCAGGGCATGGTGGC to detect the allele 2 sequence.

In IRAK1 The TaqMan MGB probe/extension primers were VIC AGGGGGGATGCAGCTGGCGGCCTCC to detect the allele 1 sequence and FAM AATGCCCGGGCACCCCCGC CACCAC to detect the allele 2 sequence.

The total PCR reaction volume contained $40 \mathrm{ng} / \mu \mathrm{l}$ of genomic DNA, $10 \mu \mathrm{l}$ of TaqMan master mix II (cat no. 4440043), $0.5 \mu \mathrm{l} 20 \times$ SNP assay mix and was adjusted to a final volume of $20 \mu \mathrm{l}$ using nuclease free water. The PCR was performed by Step-one real time PCR (Applied Biosystems; Singapore), under the following conditions: initial enzyme activation at $95^{\circ} \mathrm{C}$ for $10 \mathrm{~min}$, followed by 40 cycles of amplification; denaturation at $95^{\circ} \mathrm{C}$ for $15 \mathrm{~s}$, annealing/extension for $1 \mathrm{~min}$ at $60^{\circ} \mathrm{C}$. Fluorescence data collection was performed at annealing/ extension step for FAM and VIC dye.

\section{Statistical analysis}

Data were coded and entered using the statistical package SPSS version 25. Data were summarized using mean, standard deviation, median, minimum and maximum for quantitative variables and frequencies (number of cases) and relative frequencies (percentages) for categorical variables. Comparisons between quantitative variables were done using the non-parametric Kruskal-Wallis and Mann-Whitney tests. For comparing categorical data, the $\chi^{2}$ test was performed. The exact test was used instead when the expected frequency was less than 5. Genotype and allele frequencies were compared between the disease and the control groups using $\chi^{2}$ tests. Odds ratio (OR) with 95\% confidence intervals $(\mathrm{Cl})$ was calculated. Haplotype frequencies and linkage disequilibrium (LD) parameters ( $D^{\prime}$ and $r^{2}$ ) were estimated by SNP Stats (http://bioinfo.iconcologia.net/ snpstats/start.htm). P-values less than 0.05 were considered as statistically significant.

\section{Results}

\section{Clinical characteristics of the study population}

In the present study, the mean age of patients was $28.40 \pm 9.23$ years and they were 47 females and 3 males. 
Table I. Demographic features, clinical and laboratory characteristics in patients with systemic lupus erythematosus

\begin{tabular}{|lcc|}
\hline Variable & Mean \pm SD (range) & $n(\%)$ \\
\hline Age (years) & $28.40 \pm 9.23(13-51)$ & \\
\hline Gender (male : female) & & $\begin{array}{c}3 / 47 \\
(6 / 94)\end{array}$ \\
\hline Disease duration (months) & $51.52 \pm 55.58(1-240)$ & \\
\hline $24 \mathrm{~h} \mathrm{proteins}(\mathrm{mg} / \mathrm{dl})$ & $1610 \pm 1997(60-11200)$ & \\
\hline C3 $(\mathrm{mg} / \mathrm{dl})$ & $79.08 \pm 37.2(15-194)$ & \\
\hline C4 $(\mathrm{mg} / \mathrm{dl})$ & $14.86 \pm 9.34(2.70-49)$ & \\
\hline SLEDAl & $17.92 \pm 7.07(6-38)$ & \\
\hline SLICC & $0.18 \pm .39(0-1.0)$ & \\
\hline Renal biopsy & & \\
\hline Class 2 & & $9(33.3)$ \\
\hline Class 3 & & $8(29.6)$ \\
\hline Class 4 & & $10(37.0)$ \\
\hline
\end{tabular}

SLEDAI - Systemic Lupus erythematosus Disease Activity Index score, SLICC DI - Systemic Lupus International Collaborating Clinics SLICC damage index.

The age ( $27.5 \pm 8.3$ years) and gender (90 females and 10 males) of controls were comparable $(p>0.05)$. Analysis of laboratory data revealed that all patients (100\%) were positive for antinuclear antibodies (ANA), 66\% of patients were positive for anti-ds-DNA, 70\% were positive for CRP while C3 and C4 were consumed in 72\% of patients. The demographic features and clinical and laboratory characteristics of the patients are presented in Table I.
As regards the SLEDAl score in all SLE cases, it ranged from 6 to 38 with a mean of $17.92 \pm 7.07$, while the SLICC score ranged from 0 to 1 with a mean of 0.18 \pm 0.39 . A significant difference between patients with lupus nephritis and non-lupus nephritis patients regarding SLEDAI score was detected ( $p=0.001$ ), but no significant difference was detected between both subgroups of patients regarding SLICC score $(p>0.05)$.

\section{Association between MECP2 and IRAK1 gene polymorphisms and risk of SLE}

The results of the frequency distribution of MECP2 (rs1734791) and IRAK1 (rs1059703) SNP variants in both the study and control groups conformed to Hardy-Weinberg equilibrium $(p>0.05)$.

Analyses of the genotype and allele frequencies of MECP2 (rs1734791) taking the A allele as a reference allele revealed that SLE patients had a higher frequency of the homozygous AA genotype in comparison to healthy controls ( $50 \%$ vs. $30 \%$ respectively), and lower frequency of the homozygous TT genotype in comparison to healthy controls ( $8 \%$ vs. $40 \%$ respectively, $O R=0.120$, $95 \% \mathrm{Cl}=0.038-0.382)$ and this association was significant $(p<0.001)$. AT + TT carriers represent $50 \%$ of SLE patients and $70 \%$ of controls. This association is significant in codominant, dominant and recessive models of inheritance $(p<0.05)$. The frequency of the T allele was $29.0 \%$ in SLE patients and $55.0 \%$ in the healthy control group while the frequency of the A allele was $71.0 \%$ in SLE patients and $45.0 \%$ in the healthy control group $(\mathrm{OR}=0.334,95 \% \mathrm{Cl}=0.2-0.559, p<0.001)$ (Table II).

Table II. MECP2 (rs1734791) gene polymorphisms in systemic lupus erythematosus patients and controls

\begin{tabular}{|c|c|c|c|c|c|c|c|c|c|}
\hline \multirow[t]{2}{*}{ Model } & & \multicolumn{2}{|c|}{ Cases } & \multicolumn{2}{|c|}{ Controls } & \multirow[t]{2}{*}{$p$-value } & \multirow[t]{2}{*}{ OR } & \multicolumn{2}{|c|}{$95 \% \mathrm{Cl}$} \\
\hline & & $n$ & $\%$ & $n$ & $\%$ & & & Lower & Upper \\
\hline \multirow[t]{7}{*}{ Codominant } & Genotypes & & & & & & & & \\
\hline & $A A$ & 25 & 50 & 30 & 30 & & & & \\
\hline & AT & 21 & 42 & 30 & 30 & $<0.001^{*}$ & 0.840 & 0.389 & 1.814 \\
\hline & TT & 4 & 8 & 40 & 40 & & 0.120 & 0.038 & 0.382 \\
\hline & Alleles & & & & & & & & \\
\hline & A & 71 & 71 & 90 & 45 & & & & \\
\hline & $T$ & 29 & 29 & 110 & 55 & $<0.001^{\star}$ & 0.334 & 0.20 & 0.559 \\
\hline \multirow[t]{2}{*}{ Dominant } & $A / A$ & 25 & 50 & 30 & 30 & & 1 & & \\
\hline & $\mathrm{A} / \mathrm{T}-\mathrm{T} / \mathrm{T}$ & 25 & 50 & 70 & 70 & $0.017^{\star}$ & 0.43 & 0.21 & 0.86 \\
\hline \multirow[t]{2}{*}{ Recessive } & $\mathrm{A} / \mathrm{A}-\mathrm{A} / \mathrm{T}$ & 46 & 92 & 60 & 60 & & 1 & & \\
\hline & $T / T$ & 4 & 8 & 40 & 40 & $<0.0001^{*}$ & 0.13 & 0.04 & 0.39 \\
\hline \multirow[t]{2}{*}{ Overdominant } & $\mathrm{A} / \mathrm{A}-\mathrm{T} / \mathrm{T}$ & 29 & 58 & 70 & 70 & & & & \\
\hline & $A / T$ & 21 & 42 & 30 & 30 & 0.15 & 1.69 & 0.83 & 3.42 \\
\hline
\end{tabular}

* Significance difference $(p<0.05), \mathrm{Cl}$ - confidence interval, OR - odds ratio. 
Table III. IRAK1(rs1059703) gene polymorphisms in systemic lupus erythematosus patients and controls

\begin{tabular}{|c|c|c|c|c|c|c|c|c|c|}
\hline \multirow[t]{2}{*}{ Model } & & \multicolumn{2}{|c|}{ Cases } & \multicolumn{2}{|c|}{ Controls } & \multirow[t]{2}{*}{$p$-value } & \multirow[t]{2}{*}{ OR } & \multicolumn{2}{|c|}{$95 \% \mathrm{Cl}$} \\
\hline & & $n$ & $\%$ & $n$ & $\%$ & & & Lower & Upper \\
\hline \multirow[t]{7}{*}{ Codominant } & Genotypes & & & & & & & & \\
\hline & GG & 4 & 8.0 & 30 & 30.0 & & 1 & & \\
\hline & $A G$ & 21 & 42.0 & 25 & 25.0 & $0.0029^{*}$ & 6.30 & 1.91 & 20.79 \\
\hline & AA & 25 & 50.0 & 45 & 45.0 & & 4.17 & 1.32 & 13.19 \\
\hline & Alleles & & & & & & & & \\
\hline & G & 29 & 29.0 & 84 & 42.0 & & & & \\
\hline & A & 71 & 71.0 & 116 & 58.0 & $0.029^{\star}$ & 1.773 & 1.059 & 2.968 \\
\hline \multirow[t]{2}{*}{ Dominant } & $\mathrm{G} / \mathrm{G}$ & 4 & 8.0 & 30 & 30.0 & $0.0012^{*}$ & & & \\
\hline & $\mathrm{A} / \mathrm{G}-\mathrm{A} / \mathrm{A}$ & 46 & 92 & 70 & 70 & & 4.93 & 1.63 & 14.92 \\
\hline \multirow[t]{2}{*}{ Recessive } & $\mathrm{G} / \mathrm{G}-\mathrm{A} / \mathrm{G}$ & 25 & 50 & 55 & 55 & 0.56 & & & \\
\hline & $A / A$ & 25 & 50 & 45 & 45 & & 1.22 & 0.62 & 2.41 \\
\hline \multirow[t]{2}{*}{ Overdominant } & $\mathrm{A} / \mathrm{A}-\mathrm{G} / \mathrm{G}$ & 29 & 58 & 75 & 70 & 1 & & & \\
\hline & $\mathrm{A} / \mathrm{G}$ & 21 & 42 & 25 & 30 & $0.035^{\star}$ & 2.17 & 1.06 & 4.47 \\
\hline
\end{tabular}

* Significance difference $(p<0.05), C l$ - confidence interval, OR - odds ratio.

Analyses of the genotype and allele frequencies of IRAK1 (rs1059703) SNP taking the G allele as a reference allele revealed that SLE patients had a lower frequency of the homozygous GG genotype in comparison to healthy controls ( $8 \%$ vs. $30 \%$ respectively), and a higher frequency of the heterozygous $A G$ and homozygous AA genotypes in comparison to healthy controls (42\% vs. $25 \%$ respectively, $\mathrm{OR}=6.30,95 \% \mathrm{Cl}=1.91-20.79$ and $50 \%$ vs. $45 \%$ respectively, $\mathrm{OR}=4.17,95 \% \mathrm{Cl}=1.32-3.19$ ); these associations were significant ( $p=0.0029)$. AG+ AA carriers represent $92 \%$ of SLE and $70 \%$ of controls. This association is significant in codominant, dominant and overdominant models of inheritance $(p<0.05)$ The frequency of the A allele was $71.0 \%$ in SLE patients and $58 \%$ in the healthy control group (OR $=1.773,95 \%$ $\mathrm{Cl}=1.059-2.968, p=0.029$ ) (Table III).

\section{Genetic effects of haplotypes of the MECP2 and IRAK1 gene on SLE risk}

Haplotype analysis revealed that the haplotype $T+G$ of rs1734791 and rs1059703 SNPS is a protective haplotype with a frequency of $26 \%$ in SLE patients compared to $39.6 \%$ in healthy controls (OR $=0.47, p=0.0046)$. Also haplotype T+A of rs1734791 and rs1059703 SNPS is a protective haplotype with a frequency of $0.3 \%$ in SLE patients and $15.3 \%$ in healthy controls $(O R=0.3$, $p<0.012$ ) while $A+A$ and $A+G$ haplotypes were more frequent in SLE patients (Table IV).

The haplotype estimates $\left(D^{\prime}=0.8562 ; r^{2}=0.7214\right.$; $p<0.001)$ indicate the presence of a level of LD between
MECP2 (rs1734791) and IRAK1 (rs1059703) SNPs in the Egyptian population.

\section{MECP2 and IRAK1 genetic variants and SLE phenotype}

Studying the genetic influence of rs1734791 and rs1059703 on clinical and laboratory parameters revealed no significant difference among different genotypes of both SNPS regarding laboratory parameters except for C4, which was significantly consumed in SLE patients carrying AA genotype of IRAK1 (rs1059703) in comparison to carriers of GG genotype $(p=0.019)$. Otherwise no significant difference was detected regarding other laboratory data (data not shown), clinical parameters or between lupus-nephritis patients and non-lupus-nephritis patients regarding genotype frequencies of both SNPS $(p>0.05)$ (Table V).

On studying disease activity according to SLEDAI score, mild, moderate and severe grades of disease activity show no significant difference regarding the distribution of genotype frequencies of both SNPS. Also studying disease severity according to SLICC score did not reveal any significant difference among SLE patients carrying different genotypes of both SNPS ( $p$-value $>0.05)$.

\section{Discussion}

A complex interaction between genetic and epigenetic factors was implicated in the pathogenesis of 
Table IV. MECP2 (rs1734791) and IRAK1(rs1059703) haplotype frequencies in SLE patients and controls

\begin{tabular}{|ccccccc|}
\hline MECP2 & IRAK1 & \multicolumn{3}{c}{ Frequency } & OR $(95 \% \mathrm{Cl})$ & $p$-value \\
\cline { 3 - 5 } & & Total & Cases & Controls & & \\
\hline A & A & 0.5075 & 0.679 & 0.4219 & 1.00 & $0.0046^{*}$ \\
\hline T & G & 0.3508 & 0.259 & 0.3969 & $0.47(0.28-0.79)$ & $0.012^{*}$ \\
\hline T & A & 0.1125 & 0.031 & 0.1531 & $0.30(0.12-0.76)$ & 0.99 \\
\hline A & G & 0.0292 & 0.031 & 0.0281 & $0.99(0.26-3.76)$ & \\
\hline
\end{tabular}

"Significance difference $(p<0.05), \mathrm{Cl}$ - confidence interval, OR - odds ratio.

Table V. Association between clinical data and MECP2 (rs1734791) and IRAK1 (rs1059703) SNPs in SLE patients (dominant model)

\begin{tabular}{|c|c|c|c|c|c|c|c|c|c|c|}
\hline \multirow[t]{3}{*}{ Characteristic } & \multicolumn{5}{|c|}{ MECP2 } & \multicolumn{5}{|c|}{ IRAK1 } \\
\hline & \multicolumn{2}{|c|}{$A A$} & \multicolumn{2}{|c|}{ Non-AA } & \multirow[t]{2}{*}{$p$-value } & \multicolumn{2}{|c|}{ GG } & \multicolumn{2}{|c|}{ Non-GG } & \multirow[t]{2}{*}{$p$-value } \\
\hline & $n$ & $\%$ & $n$ & $\%$ & & $n$ & $\%$ & $n$ & $\%$ & \\
\hline Malar rash & 24 & 51.1 & 23 & 48.9 & 0.5 & 4 & 8.5 & 43 & 91.5 & 0.77 \\
\hline Discoid rash & 1 & 20 & 4 & 80 & 0.17 & 0 & 0 & 5 & 100 & 0.647 \\
\hline Photosensitivity & 19 & 50 & 19 & 50 & 0.629 & 4 & 10.5 & 34 & 89.5 & 0.321 \\
\hline Oral ulcers & 12 & 42.9 & 16 & 57.1 & 0.197 & 4 & 14.3 & 24 & 85.7 & 0.089 \\
\hline Arthritis & 16 & 61.5 & 10 & 38.5 & 0.078 & 1 & 3.8 & 25 & 96.2 & 0.275 \\
\hline Renal disorder & 12 & 44.4 & 15 & 55.6 & 0.285 & 2 & 7.4 & 25 & 92.6 & 0.632 \\
\hline Hematological & 8 & 47.1 & 9 & 52.9 & 0.5 & 0 & 0 & 17 & 100 & 0.178 \\
\hline Serositis & 11 & 64.7 & 6 & 35.3 & 0.116 & 0 & 0 & 17 & 100 & 0.178 \\
\hline Alopecia & 10 & 52.6 & 9 & 47.4 & 0.5 & 0 & 0 & 19 & 100 & 0.137 \\
\hline Myositis & 25 & 50 & 25 & 50 & 0.16 & 4 & 8 & 46 & 92.0 & 0.21 \\
\hline Fever & 5 & 55.6 & 4 & 44.4 & 0.50 & 0 & 0 & 9 & 100 & 0.44 \\
\hline Neurologic & 1 & 0 & 4 & 80 & 0.174 & 0 & 0 & 5 & 100 & 0.647 \\
\hline Vasculitis & 2 & 66.7 & 1 & 33.3 & 0.50 & 0 & 0 & 3 & 100 & 0.774 \\
\hline
\end{tabular}

lupus [17]. Alteration of DNA methylation pattern of T cells by DNA methylation inhibitor enhances expression of methylation sensitive genes, and induces in vitro autoreactivity, glomerulonephritis and a lupuslike disease in an animal model [11].

MECP2 is a multifunctional epigenetic regulator that can act as a transcriptional repressor and activator according to the methylation state of the targeted gene and interactions of MECP2 with either inhibitors or activators of transcription [18]. MECP2 gene polymorphism was associated with the pathogenesis of SLE in a few studies, yet risk alleles of different SNPS of MECP2 gene are controversial in different studies and populations.

In our study in the Egyptian population, examination of MECP2 SNP (rs1734791) revealed that the A allele was the risk allele for lupus as it was more pronounced in lupus patients and the difference between patients and controls was significant, while the T allele and TT genotype frequencies were significantly higher in healthy controls. Our results were in accordance with the results of two previous studies conducted on two different populations. One study was conducted by Sawalha et al. [11], who identified the A allele of the MECP2 SNP (rs1734791) as a lupus risk allele in two different SLE cohorts from Korean and European populations.

The other was conducted by Webb et al. [12], on a larger cohort of European populations and they formed the same conclusions. Furthermore, these authors conducted a functional study to examine the effect of both disease-risk and protective haplotypes on the expression patterns of MECP2 regulated genes derived from B cell lines of European female lupus patients. They found that in females with the risk haplotype $81 \%$ of targeted genes were upregulated while 19\% were downregulated. They also found that lupus-risk MECP2 haplotype is associated with higher expression of CREB1 (a transcriptional co-activator) and lower expression of HDAC1 (a transcriptional co-repressor) [12]. 
In contrast to our results, the T allele of the rs1734791 SNP was identified as a lupus risk allele in two other studies. One of them was conducted by Alesaeidi et al. [13] on the Iranian population; however they also reported no significant association between the genotype frequency of this SNP and the risk of SLE and no correlation of this SNP with clinical manifestations of SLE

The other study was conducted by Kaufman et al. [8] on SLE subjects derived from four different ancestral groups. Although the T allele of the rs1734791 SNP was significantly associated with the risk of SLE in their study, they demonstrated that lower levels of MECP2 mRNA that resulted in dysregulation of SLE-risk gene expression in patients and controls was associated with the SLE-risk genotype of the rs1059702 SNP of the IRAK1 gene

Due to the strong linkage disequilibrium between IRAK1 and MECP2 genes, we also examined rs1059703 on the IRAK1 gene. The variant of this SNP that results in a substitution of leucine by serine amino acid at position 532 was demonstrated to have a higher level of autophosphorylation and increased NF- $\mathrm{KB}$ activity in vitro than the wild type [19]. Our results on the Egyptian population revealed that GG genotype was more frequently represented in controls than SLE patients while AA and AG genotypes and the A allele were more frequently represented in SLE patients than controls and these differences were significant.

In the study of Kaufman et al. [8], rs1059703 was associated with SLE in European American (EA), Hispanic (HA) and Asian (AS) subjects but no significant association was found in patients with African ancestry. Moreover, they attributed the association of rs1059703 with SLE in EA, AS and HA to rs1059702 because rs1059703 and rs1059702 were in strong LD in these populations but in low LD in patients with African ancestry.

IRAK1 and MECP2 regulate the immune response and also regulate each other at multiple levels to ensure immune hemostasis at steady state. IRAK1 activates NF-кB in response to Toll-like receptor/interleukin 1 receptor ligands; hence IRAK1 upregulates inflammatory genes' expression [20]. This is supported by the fact that IRAK1-deficient mice are less prone to autoimmunity; moreover, TGF- $\beta$ induction of Foxp3+ Tregs is more efficient in Irak1-deficient CD4+ T cells [21].

Following MECP2 loss of function, IRAK1 is upregulated, resulting in NF-אB pathway upregulation [22], MECP2 counteracting epigenetic silencing of Treg Foxp3 that is induced by inflammation, and deletion of MECP2 in mice Tregs causes spontaneous immune activation [23]. Also MECP2 expression is partially regulated by signaling pathways involving NF-кB [24]. Furthermore, the role of the environment is important, as in genetically predisposed individuals, environmental exposures may epigenetically modify SLE risk genes, causing the development of SLE manifestations [25]. We can realize the complexity of interplay between both genes when a certain genetic variant influenced by specific environmental triggers may tilt the balance towards inflammatory or anti-inflammatory phenotype in a certain population.

In our study the classical complement pathway component C4 was significantly consumed in SLE patients carrying AA genotype of IRAK1 (rs1059703) SNP in comparison to carriers of GG genotype. The complement system plays a role in removal of apoptotic cells and immune complexes and deficiency of the early complement components of the classical pathway "C1q, C4, and C2" is a risk factor for developing SLE [26].

Song [27] reported crosstalk between TLRs (which act upstream of the IRAK1 signaling pathway) and complement and that crosstalk can occur both ways. While the complement anaphylatoxins augmented TLR-induced proinflammatory cytokine production, TLR activation could also increase complement protein synthesis and function. Thus whether AA genotype of IRAK1 (rs1059703) SNP upregulates both pathways to give significant positive feedback on the inflammatory response and plays a role in the pathogenesis of the disease needs future intense research.

\section{Conclusions}

There is a possible genetic association between both MECP2 (rs1734791) (A > T) and IRAK1 (rs1059703) (G > A) SNP and susceptibility to SLE, while no significant association between either SNP and disease activity or severity was detected.

This research did not receive any specific grant from funding agencies in the public, commercial, or not-forprofit sectors.

The authors declare no conflict of interest.

\section{References}

1. Hedrich CM. Epigenetics in SLE. Curr Rheumatol Rep 2017; 19: 58.

2. Miller FW, Alfredsson L, Costenbader KH, et al. Epidemiology of environmental exposures and human autoimmune diseases: findings from a National Institute of Environmental Health Sciences Expert Panel Workshop. J Autoimmun 2012; 39: 259-271.

3. Hedrich CM, Crispin JC, Tsokos GC. Epigenetic regulation of cytokine expression in systemic lupus erythematosus with special focus on T cells. Autoimmunity 2014; 47: 234-241.

4. Hedrich CM, Mabert K, Rauen T, Tsokos GC. DNA methylation in systemic lupus erythematosus. Epigenomics 2017; 9: 505-525. 
5. Javierre BM, Fernandez AF, Richter J, et al. Changes in the pattern of DNA methylation associate with twin discordance in systemic lupus erythematosus. Genome Res 2010; 20: 170-179.

6. Koelsch KA, Webb R, Jeffries M, et al. Functional characterization of the MECP2/IRAK1 lupus risk haplotype in human T cells and a human MECP2 transgenic mouse. J Autoimmun 2013; 41: 168-174.

7. Young J, Hong EP, Castle JC, et al. Regulation of RNA splicing by the methylation-dependent transcriptional repressor methylCpG binding protein 2. Proc Natl Acad Sci U S A 2005; 102 17551-17558.

8. Kaufman KM, Zhao J, Kelly JA, et al. Fine Mapping of Xq28: Both MECP2 and IRAK1 Contribute to Risk for Systemic Lupus Erythematosus in Multiple Ancestral Groups. Ann Rheum Dis 2013; 72: 437-444.

9. Hartupee J, Li X, Hamilton T. Interleukin 1alpha-induced NF kappa $B$ activation and chemokine mRNA stabilization diverge at IRAK1. J Biol Chem 2008; 283: 15689-15693.

10. Marshak-Rothstein A. Toll-like receptors in systemic autoimmune disease. Nat Rev Immunol 2006; 6: 823-835.

11. Sawalha AM, Webb R, Han S, et al. Common Variants within MECP2 Confer Risk of Systemic Lupus Erythematosus. PLoS One 2008; 3: e1727.

12. Webb R, Wren JD, Jeffries $M$, et al. Variants within MECP2, a key transcriptional regulator, are associated with increased sus ceptibility to lupus and differential gene expression in lupus patients. Arthritis Rheum 2009; 60: 1076-1084.

13. Alesaeidi S, Karami J, Mahmoudi M, et al. Methyl-CpG-Binding Protein 2 (MECP2) Polymorphism in Iranian Patients with Systemic Lupus Erythematosus. Inflammation 2015; 38: 2185 2190.

14. Petri M, Orbai AM, Alarcón GS, et al. Derivation and validation of the Systemic Lupus International Collaborating Clinics classification criteria for systemic lupus erythematosus. Arthritis Rheum 2012; 64: 2677-2686.

15. Gladman DD, Ibañez D, Urowitz MB. Systemic lupus erythematosus disease activity index. J Rheumatol 2002; 29: 288-291.
16. Gladman D, Ginzler E, Goldsmith C, et al. The development and initial validation of the Systemic Lupus International Collaborating Clinics/American College of Rheumatology damage index for systemic lupus erythematosus. Arthritis Rheum 1996; 39: 363-369.

17. Deng Y, Tsao BP. Advances in lupus genetics and epigenetics. Curr Opin Rheumatol 2014; 26: 482-492.

18. Della Ragione F, Vacca M, Fioriniello S, et al. MECP2, a multi-talented modulator of chromatin architecture. Brief Funct Genomics 2016; 15: 420-431.

19. Liu G, Tsuruta Y, Gao Z, et al. Variant IL-1 receptor-associated kinase-1 mediates increased NF-kappa B activity. J Immunol 2007; 179: 4125-4134.

20. Liu G, Park YJ, Abraham E. Interleukin-1 receptor-associated kinase (IRAK) -1-mediated NF-kappaB activation requires cytosolic and nuclear activity. FASEB J 2008; 22: 2285-2296.

21. Heiseke AF, Jeuk BH, Markota A, et al. IRAK1 Drives Intestinal Inflammation by Promoting the Generation of Effector Th Cells with Optimal Gut-Homing Capacity. J Immunol 2015; 195: 5787-5794.

22. Kishi N, MacDonald JL, Ye J, et al. Reduction of aberrant NF- $\kappa B$ signalling ameliorates Rett syndrome phenotypes in Mecp2null mice. Nat Commun 2016; 7: 10520.

23. Li C, Jiang S, Liu SQ, et al. MeCP2 enforces Foxp3 expression to promote regulatory $T$ cells' resilience to inflammation. Proc Natl Acad Sci USA 2014; 111: E2807-2816.

24. O’Driscoll C, Kaufmann WE, Bressler J. Relationship between Mecp2 and NFkb signaling during neural differentiation of P19 cells. Brain Res 2013; 1490: 35-42.

25. Barbhaiya M, Costenbader KH. Environmental Exposures and the Development of Systemic Lupus Erythematosus. Curr Opin Rheumatol 2016; 28: 497-505.

26. Walport MJ. Complement. N Engl J Med 2001; 344: 1058-1066.

27. Song WC. Crosstalk between Complement and Toll-Like Receptors. Toxicol Pathol 2012; 40: 174-182. 